\title{
A NOTE ON HARDY LIKE INTEGRAL INEQUALITIES
}

\author{
B.G. PACHPATTE
}

\begin{abstract}
The aim of the present note is to establish two new integral inequalities of the Hardy type by using a fairly elementary analysis.
\end{abstract}

\section{Introduction}

IN [3] G.H. Hardy proved the following integral inequality. If $p>1, f(x) \geq 0$ for $0<x<\infty$ and $F(x)=\int_{0}^{x} f(t) d t$, then

$$
\int_{0}^{\infty}\left(\frac{F}{x}\right)^{p} d x<\left(\frac{p}{p-1}\right)^{p} \int_{0}^{\infty} f^{p} d x
$$

unless $f(x)$ is identically zero.

This inequality has received wide attention after the book "Inequalities" written in 1934 by Hardy, Littlewood and Polya [4, p. 240]. There exists a vast literature which deals with the simple proofs, various generalizations and series analogues of Hardy's inequality (1) and its generalizations in several different directions, see [2, 6-9] and the references given therein. Our objective here is to establish two new integral inequalities which in the special cases reduces to the inequalities similar to that of the variants of Hardy's inequality (1) given by Adams [1, p. 402], Zygmund [10, p. 20] and Izumi and Izumi [5, p. 279]. An interesting feature of the inequalities established here is that the analysis used in their proofs is quite elementary and our results provide new estimates on inequalities of this type. Throughout the presesnt note we shall assume that all the integrals exists on the respective domains of their definitions.

\section{Statement of Results}

Our main result is given in the following theorem.

Received September 30, 1992; revised December 16, 1993.

1991 Mathematics Subject Classification. Primary 26D15, Secondary 26D20.

Key words and phoses. Integral inequalities, Hardy type, integrable function, Hölder's inequality. 
Theorem 1. Let $\alpha \geq 0, p \geq 0, q \geq 1$ be real constants, $f$ be a nonnegative and integrable function on $(0, a)$ for fixd $a>0$. If $F(x)=\int_{0}^{x} f(t) d t$, then

$$
\int_{0}^{a} x^{\alpha} F^{p+q}(x) d x \leq M^{q} \int_{0}^{a} x^{\alpha+q} F^{p}(x)\left\{\frac{F(x)}{a}+f(x)\right\}^{q} d x,
$$

where $M=\max \left\{\frac{\alpha+2}{\alpha+1}, \frac{2(p+q)}{\alpha+1}\right\}$.

A slightly different version of Theorem 1 is established in the following theorem.

Theorem 2. Let $\alpha, p, q, f$ be as defined in Theorem 1. If $G(x)=\int_{\frac{x}{2}}^{x} f(t) d t$, then

$$
\int_{0}^{a} x^{\alpha} G^{p+q}(x) d x \leq M^{q} \int_{0}^{a} x^{\alpha+q} G^{p}(x)\left\{\frac{G(x)}{a}+\left|f(x)-\frac{1}{2} f\left(\frac{x}{2}\right)\right|\right\}^{q} d x,
$$

where $M$ is as defined in Theorem 1.

Remark 1. We note that in the special case when $p=0$, inequalities (2) and (3) reduce respectively to the following inequalities

$$
\begin{gathered}
\int_{0}^{a} x^{\alpha} F^{q}(x) d x \leq M_{0}^{q} \int_{0}^{a} x^{\alpha+q}\left\{\frac{F(x)}{a}+f(x)\right\}^{q} d x, \\
\int_{0}^{a} x^{\alpha} G^{q}(x) d x \leq M_{0}^{q} \int_{0}^{a} x^{\alpha+q}\left\{\frac{G(x)}{a}+\left|f(x)-\frac{1}{2} f\left(\frac{x}{2}\right)\right|\right\}^{q} d x,
\end{gathered}
$$

where $M_{0}=\max \left\{\frac{\alpha+2}{\alpha+1}, \frac{2 q}{\alpha+1}\right\}$. It is easy to observe that the inequalities obtained in (4) and (5) differ from those of given by various authors in [1-10].

\section{Proofs of Theorems 1 and 2}

If $f$ is null, then inequality (2) in Theorem 1 is trivially true. We assume that $f$ is not null. Integrating by parts, we have the following identity:

$$
\begin{aligned}
& \int_{0}^{a}\left[x^{\alpha+1}-\frac{1}{a} x^{\alpha+2}\right] F^{p+q-1}(x) f(x) d x \\
= & -\int_{0}^{a}\left[(\alpha+1) x^{\alpha}-\frac{1}{a}(\alpha+2) x^{\alpha+1}\right] \frac{F^{p+q}(x)}{p+q} d x .
\end{aligned}
$$

From (6) we observe that

$$
\begin{aligned}
\int_{0}^{a} x^{\alpha} F^{p+q}(x) d x= & \frac{(\alpha+2)}{(\alpha+1) a} \int_{0}^{a} x^{\alpha+1} F^{p+q}(x) d x \\
& -\frac{(p+q)}{(\alpha+1)} \int_{0}^{a}\left[x^{\alpha+1}-\frac{1}{a} x^{\alpha+2}\right] F^{p+q-1}(x) f(x) d x
\end{aligned}
$$




$$
\begin{aligned}
& \leq \frac{(\alpha+2)}{(\alpha+1) a} \int_{0}^{a} x^{\alpha+1} F^{p+q}(x) d x \\
& +\frac{(p+q)}{(\alpha+1)} \int_{0}^{a} x^{\alpha+1}\left[1+\frac{1}{a} x\right] F^{p+q-1}(x) f(x) d x \\
& \leq \frac{(\alpha+2)}{(\alpha+1) a} \int_{0}^{a} x^{\alpha+1} F^{p+q}(x) d x \\
& +\frac{2(p+q)}{(\alpha+1)} \int_{0}^{a} x^{\alpha+1} F^{p+q-1}(x) f(x) d x \\
& \leq M \int_{0}^{a} x^{\alpha+1} F^{p+q-1}(x)\left\{\frac{F(x)}{a}+f(x)\right\} d x \\
& =M \int_{0}^{a}\left[x^{\alpha+1-\alpha\left(\frac{q-1}{q}\right)} F^{\frac{p}{q}}(x)\left\{\frac{F(x)}{a}+f(x)\right\}\right] \\
& \cdot\left[x^{\alpha\left(\frac{q-1}{q}\right)} F^{p+q-1-\frac{p}{q}}(x)\right] d x .
\end{aligned}
$$

By using the Hölder's inequality with indices $q, \frac{q}{q-1}$ on the right side of (7) we get

$$
\begin{aligned}
\int_{0}^{a} x^{\alpha} F^{p+q}(x) d x & \leq M\left\{\int_{0}^{a} x^{\alpha+q} F^{p}(x)\left\{\frac{F(x)}{a}+f(x)\right\}^{q} d x\right\}^{\frac{1}{q}} \\
& \cdot\left\{\int_{0}^{a} x^{\alpha} F^{p+q}(x) d x\right\}^{\frac{q-1}{q}} .
\end{aligned}
$$

Now dividing both sides of (8) by $\left\{\int_{0}^{a} x^{\alpha} F^{p+q}(x) d x\right\}^{\frac{q-1}{q}}$ and then raising the $q$ th power on both sides of the resulting inequality, we get the required inequality in (2). This completes the proof of Theorem 1 .

In order to establish the inequality (3) in Theorem 2, we assume as in the proof of Theorem 1 , the function $f$ is not null. Integrating by parts, we have the followin identity:

$$
\begin{aligned}
& \int_{0}^{a}\left[x^{\alpha+1}-\frac{1}{a} x^{\alpha+2}\right] G^{p+q-1}(x)\left[f(x)-\frac{1}{2} f\left(\frac{x}{2}\right)\right] d x \\
= & -\int_{0}^{a}\left[(\alpha+1) x^{\alpha}-\frac{1}{a}(\alpha+2) x^{\alpha+1}\right] \frac{G^{p+q}(x)}{p+q} d x .
\end{aligned}
$$

Now by following exactly the same arguments as in the proof of Theorem 1 given below the identity (6) with suitable modifications, we get the desired inequality in (3). The proof of Theorem 2 is complete.

Remark 2. It is interesting to note that if the function $f$ involved in (2)-(5) is integrable on $(0, \infty)$, then letting $a \rightarrow \infty$ in (2)-(5) we get respectively the following inequalities

$$
\begin{gathered}
\int_{0}^{\infty} x^{\alpha} F^{p+q}(x) d x \leq M^{q} \int_{0}^{\infty} x^{\alpha+q} F^{p}(x) f^{q}(x) d x \\
\int_{0}^{\infty} x^{\alpha} G^{p+q}(x) d x \leq M^{q} \int_{0}^{\infty} x^{\alpha+q} G^{p}(x)\left|f(x)-\frac{1}{2} f\left(\frac{x}{2}\right)\right|^{q} d x
\end{gathered}
$$




$$
\begin{gathered}
\int_{0}^{\infty} x^{\alpha} F^{q}(x) d x \leq M_{0}^{q} \int_{0}^{\infty} x^{\alpha}(x f(x))^{q} d x \\
\int_{0}^{\infty} x^{\alpha} G^{q}(x) d x \leq M_{0}^{q} \int_{0}^{\infty} x^{\alpha+q}\left|f(x)-\frac{1}{2} f\left(\frac{x}{2}\right)\right|^{q} d x
\end{gathered}
$$

where $M$ and $M_{0}$ are as defined in Theorem 1 and Remark 1 . Here it is to be noted that the inequalities obtained in (12) and (13) are respectively similar to those of the variants of Hardy's inequality given by Zygmund [10, p.20] and Izumi and Izumi [5, p.279].

\section{References}

[1] R.A. Adarns, "Some integral inequalities with applications to the imbedding of Sobolev spaces defined over irregular domains," Trans. Amer. Math., Soc. 178 (1973), 401-429.

[2] P.R. Beesack, "Integral inequalities involving a function and its derivative," Amer. Math., Monthly, 78 (1971), 705-741.

[3] G.H. Hardy, "Note on a theorem of Hilbert," Math. Z., 6 (1920), 314-317.

[4] G.H. Hardy, J.E. Littlewood and G. Polya, Inequalities, Cambridge Univ., Press, Cambridge, 1934.

[5] M. Izumi and S. Izumi, "On some inequalities for Fourier Series," J. Analyse Math., 21 (1968), 277-291.

[6] P.D. Johnson Jr and R.N. Mohapatra, "Inequalities involving lowertrangular matrices," Proc. London Math., Soc. 41 (1980), 83-137.

[7] B.G. Pachpatte, "On a new class of Hardy type inequalities," Proc. Royal Soc., Edinburgh, 105A (1987), 265-274.

[8] B.G. Pachpatte, "On some variants of Hardy's inequality," J. Math. Anal. Appl., 124(1987), 495501.

[9] B.G. Pachpatte, "On some integral inequalities similar to Hardy's inequality," J. Math. Anal. Appl., 129(1988), 596-606.

[10] A. Zygmund, Trigonometrical series, Vol. I, 2nd edition, Cambridge Univ., Press, New York, 1959. 